\title{
Isolation and Probiotic Potential of Lactobacillus salivarius and Pediococcus pentosaceus in Specific Pathogen Free Chickens
}

\section{-Author(s)}

\section{Chen $\mathrm{F}^{\prime}$ \\ Zhu L'}

Qiu H"

Institute of Animal Nutritional and Metabolic Disorders, College of Animal Science and Veterinary Medicine, Qingdao Agricultural University, Qingdao, 266109, China

" Haidu College, Qingdao Agricultural University, Laiyang, 265200, China

\section{-Mail Address}

Corresponding author e-mail address Huiling Qiu

Haidu College, Qingdao Agricultural University, Laiyang, 265200, China

Tel: $\quad$ +86-535-2922076

Email: hlqiu_njau@163.com

\section{-Keywords}

Lactobacillus salivarius, Pediococcus pentosaceus, probiotic potential, specific pathogen free chicken.

\section{ABSTRACT}

This study was conducted to isolate Lactobacillus salivarius and Pediococcus pentosaceus strains from cecal content and investigate their probiotic potential in specific pathogen free (SPF) chickens. $L$. salivarius and $P$. pentosaceus strains were isolated from the cecal content of SPF chickens and identified by $16 \mathrm{~s}$ rDNA sequence analysis by BLAST analysis at the National Center for Biotechnology Information and phylogenetic analysis using DNAStar software. In an in vivo experiment, 180 7-day-old SPF chickens were randomly assigned into three groups. Group 1 served as a control that was fed a basal diet without probiotic supplementation, and groups 2 and 3 were fed the basal diets supplemented with $L$. salivarius and $P$. pentosaceus at $2 \times 10^{8} \mathrm{CFU} / \mathrm{g}$, respectively. Body weight (BW), average daily gain $(A D G)$, feed conversion ratio (FCR), dressing percentage (DP), and the apparent digestibility of crude protein (AD-CP) were calculated. We also determined meat color, fat content, shear force, water content and $\mathrm{pH}$ value of breast and thigh muscles; ammonia, urea nitrogen and uric acid content in plasma; fecal ammonia emission level and $\mathrm{pH}$ value; and Lactobacillus and Escherichia coli in ceca. Compared with the control group, $L$. salivarius and $P$. pentosaceus supplementation significantly increased $B W, A D G, D P, A D-C P$, fat content of meat, and the number of Lactobacillus in ceca $(p<0.05)$, and decreased FCR, plasma ammonia content, fecal ammonia emission, and $\mathrm{pH}$ value and the number of $E$. coli in ceca $(p<0.05)$. In the in vitro experiment, $L$. salivarius and $P$. pentosaceus treatments significantly decreased the ammonia content in medium compared with the control group without probiotic treatment $(p<0.05)$. These results suggest that $P$. pentosaceus and $L$. salivarius strains show promising probiotic properties for improving growth, meat quality and microenvironment in chickens and decreasing ammonia content in the medium.

\section{INTRODUCTION}

Probiotics such as Lactobacillus, Pediococcus, and Bifidobacterium have been defined as "a live microbial feed supplement which beneficially affects the host by improving its intestinal microbial balance" and widely distributed in nature, vegetables, dairy products, and the gastrointestinal tract of humans and animals (Carraro et al., 2011; Vidhyasagar et al., 2013). Increasing evidence indicates that Lactobacillus and Pediococcus strains exert beneficial effects by a variety of complementary mechanisms, including an ability to modulate host immune function, high adhesion capacity, cancerpreventing attributes, reduction of serum cholesterol levels, high antimicrobial activity against pathogens, and normalization of the microbiota composition (Okamoto et al., 2007; Noujaim et al., 2009; 
Meng et al., 2010; Zhang et al., 2011; Kiran et al., 2015; Xie et al., 2015; Zhang et al., 2015). Promising probiotic strains should have some superior biological properties, especially improving growth performance, intestinal microbiota balance, and meat quality, and decreasing noxious gas emission (Meng et al., 2010; Fan et al., 2013; Jeong et al., 2014; Peng et al., 2016). These have become essential indicators for evaluating the potential of food biopreservatives or probiotic additives in humans and animals (Kabir et al., 2004; Biagi et al., 2007; Nagpal et al., 2012; Mookiah et al., 2014; Altaher et al., 2015; Peng et al., 2016; Bai et al., 2017). Therefore, there is growing interest in the isolation of potentially successful probiotic strains and determination of their functional characteristics.

Pediococcus pentosaceus and Lactobacillus salivarius are most frequently isolated from the duodenal mucosa of piglets (Zhang et al., 2011; Zhang et al., 2015) and human breast milk (Osmanagaoglu et al., 2010; Kiran et al., 2015), but little information is available about the isolated strains from animals and their bioavailability. Therefore, the objectives of this study were to isolate and identify $P$. pentosaceus and $L$. salivarius strains from cecal content and investigate their potential probiotic effects on growth, meat quality and microenvironment in specific pathogen free (SPF) chickens, and ammonia treatment in vitro.

\section{MATERIAL AND METHODS}

\section{Identification of $L$. salivarius and $P$. pento- saceus strains}

The cecal content from 20-day old SPF chickens was used for isolation of lactic acid bacteria. The cecal content was serially diluted 10 -fold with phosphatebuffered saline (PBS, pH 7.4), plated on MRS agar, and incubated for $48 \mathrm{~h}$ at $37^{\circ} \mathrm{C}$ in an anaerobic incubator. Colonies were spread into MRS broth medium and incubated for $24 \mathrm{hat} 37^{\circ} \mathrm{C}$. Genomic DNA was extracted using the DNA extraction kit (TaKaRa Biotechnology, Dalian, China) and 16s rDNA gene was amplified using the primers: 5'-agagtttgatcctggctcag-3' and $5^{\prime}$-ggttaccttgttacgactt-3' and PCR kit (TaKaRa Biotechnology). PCR products were sequenced with an ABI PRISM 3730XL DNA Analyzer (Applied Biosystems, Foster (ity, (A, USA) at TaKaRa Biotechnology. Nucleotide sequences were analyzed by BLAST analysis at the National Center for Biotechnology Information (Bethesda, MD, USA), and phylogenetic tree and homology analyses were performed using the SeqEd and Cluster $V$ sequence alignment programs in the DNAStar software package (DNASTAR Inc., Wisconsin, USA). L. salivarius (QDLS-CF-01-QDLS-CF-02) and P. pentosaceus (QDPP-CF-01-QDPP-CF-04) isolates were selected and propagated at $37^{\circ} \mathrm{C}$ for $48 \mathrm{~h}$. The freeze-dried bacterial powder containing $10^{10} \mathrm{CFU} / \mathrm{g}$ was prepared and stored in sterile tubes at $4^{\circ} \mathrm{C}$.

\section{Experimental animals and diets}

One hundred and eighty 7-day-old SPF male chickens with an initial body weight (BW) of $43.5 \pm 2.2$ $g$ were purchased from Institute of Animal Husbandry and Veterinary Medicine, Shandong Academy of Agricultural Sciences and randomly allocated to three experimental treatments with three replicate pens of 20 chickens. Group 1 was fed a basal diet without probiotic supplementation, and groups 2 and 3 were fed the basal diet mixed with $L$. salivarius or $P$. pentosaceus at $2 \times 10^{7} \mathrm{CFU} / \mathrm{g}$, respectively. The basal diet was a corn-soybean meal-based diet, which was formulated to approximately meet National Research Council-recommended nutrient requirements for chickens (1994). The basal diet formulation and approximate composition are shown in Table 1. The feeding experiment lasted for 24 days and was carried out at Laboratory Animal Centre, Qingdao Agricultural University. Over the entire experimental period, water and feed were provided ad libitum, and the residual feed was collected from the cages daily.

Table 1 - Effects of Lactobacillus salivarius and Pediococcus pentosaceus supplementation on growth performance of chickens

\begin{tabular}{lccc}
\hline Group/treatment & L. salivarius & P. pentosaceus & Control \\
\hline BW $(d$ 12, g) & $125.0 \pm 5.10^{\mathrm{a}}$ & $129.7 \pm 2.05^{\mathrm{a}}$ & $107.3 \pm 3.77^{\mathrm{b}}$ \\
\hline BW $(\mathrm{d} 24, \mathrm{~g})$ & $239.8 \pm 5.68^{\mathrm{a}}$ & $256.3 \pm 10.5^{\mathrm{a}}$ & $209.9 \pm 12.1^{\mathrm{b}}$ \\
\hline ADG $(\mathrm{d} 1-12, \mathrm{~g} / \mathrm{d})$ & $7.42 \pm 0.37^{\mathrm{a}}$ & $7.73 \pm 0.15^{\mathrm{a}}$ & $5.89 \pm 0.28^{\mathrm{b}}$ \\
ADG $(\mathrm{d} 13-24, \mathrm{~g} / \mathrm{d})$ & $9.57 \pm 0.14^{\mathrm{b}}$ & $10.55 \pm 0.71^{\mathrm{a}}$ & $8.64 \pm 0.49^{\mathrm{c}}$ \\
\hline ADG (d 1-24, g/d) & $8.54 \pm 0.19^{\mathrm{b}}$ & $9.20 \pm 0.44^{\mathrm{a}}$ & $7.23 \pm 0.53^{\mathrm{c}}$ \\
\hline FCR (d 1-12) & $1.67 \pm 0.09^{\mathrm{b}}$ & $1.60 \pm 0.03^{\mathrm{b}}$ & $2.10 \pm 0.10^{\mathrm{a}}$ \\
\hline FCR (d 13-24) & $2.93 \pm 0.02^{\mathrm{b}}$ & $2.70 \pm 0.18^{\mathrm{b}}$ & $3.29 \pm 0.18^{\mathrm{a}}$ \\
\hline FCR (d 1-24) & $2.42 \pm 0.06^{\mathrm{b}}$ & $2.25 \pm 0.11^{\mathrm{b}}$ & $2.87 \pm 0.19^{\mathrm{a}}$ \\
\hline AD-CP & $46.7 \pm 0.89^{\mathrm{a}}$ & $45.2 \pm 0.67^{\mathrm{a}}$ & $41.5 \pm 0.71^{\mathrm{b}}$ \\
DP (\%) & $92.3 \pm 0.87^{\mathrm{a}}$ & $92.0 \pm 1.62^{\mathrm{a}}$ & $86.4 \pm 0.36^{\mathrm{b}}$ \\
\hline HEYP (\%) & $84.8 \pm 0.57^{\mathrm{a}}$ & $81.4 \pm 1.57^{\mathrm{b}}$ & $79.9 \pm 0.89^{\mathrm{b}}$ \\
\hline EYP (\%) & $60.6 \pm 1.07^{\mathrm{a}}$ & $58.9 \pm 1.40^{\mathrm{ab}}$ & $56.9 \pm 1.09^{\mathrm{b}}$ \\
\hline TMP (\%) & $6.03 \pm 0.58$ & $6.09 \pm 0.48$ & $5.85 \pm 0.12$ \\
\hline BMP (\%) & $4.70 \pm 0.35$ & $4.61 \pm 0.20$ & $4.20 \pm 0.37$ \\
\hline
\end{tabular}

Means in the same line with different letters were significantly different $(p<0.05)$. Data are presented as means \pm SD. 


\section{Evaluation of growth performance}

On days 12 and 24, BW and feed were recorded, and average daily gain (ADG) and feed conversion ratio (FCR) were calculated. The crude protein in feed and feces was analyzed and the apparent digestibility of crude protein (AD-CP) was calculated. On day 24, all chickens were killed with dimethyl ether. Dressing percentage (DP), the percentages of eviscerated yield (EYP) and the half eviscerated yield (HEYP), and the percentages of breast (BMP) and thigh muscle (TMP) were calculated.

\section{Evaluation of microenvironment}

On days 12 and 24, the ammonia emission level was detected by Nessler's reagent colorimetry. On day 24, anticoagulant blood samples were drawn from the heart and centrifuged at 3,000 $\times g$ for $15 \mathrm{~min}$, and plasma was collected for analysis of ammonia, uric acid and urea nitrogen (Nanjing Jiancheng Bioengineering Institute, Nanjing, China).

On day 24, 10 chickens in each replicate were randomly selected, and the ceca were removed for measuring $\mathrm{pH}$ value by using a digital $\mathrm{pH}$ meter with a $1.5-\mathrm{mm}$ microelectrode (PHS-3C; Shanghai REX Instrument Factory, China). For the measurement of cecal E. coli and Lactobacillus, the cecal contents of each chicken were thoroughly mixed in a $50 \mathrm{~mL}$ plastic centrifuge tube and diluted $10^{6}-10^{7}$ folds with PBS ( $\mathrm{pH}$ 7.4) by the multiple dilution method. Then, $1.0 \mathrm{~mL}$ aliquots were spread into Eosin Methylene Blue Agar or Lactobacillus Selection Agar plates, and incubated for $24-48 \mathrm{~h}$ at $37^{\circ} \mathrm{C}$. Colonies were counted, and the results were expressed as absolute numbers of CFU. The experiments were performed in triplicate, and the average values were calculated.

\section{Evaluation of meat quality}

On day 24, breast and thigh muscles were collected and used for detecting meat color, tenderness, fat content, water content and $\mathrm{pH}$ value within $24 \mathrm{~h}$. Meat color was evaluated by measuring total pigment content; $\mathrm{pH}$ value of muscle was measured by a digital pH meter (PH-STAR; Matthaus, Eckelsheim, Germany); tenderness was represented by shear force, which was measured by a digital muscle tenderness meter (C-LM3; Tenovo International, Beijing, China); water content was measured by the drying method; and fat content was measured by Soxhlet extractor method (Thiex et al., 2003).

\section{Evaluation of ammonia treatment in vitro}

Sixty tubes were randomly allocated to three experimental treatments, each containing 20 tubes, and each tube contained $3 \mathrm{~mL}$ PBS ( $\mathrm{pH}$ 7.4) with $0.5 \mathrm{\mu mol} / \mathrm{L}$ ammonium chloride. Group 1 was treated without probiotic supplementation, and groups 2 and 3 were treated with $L$. salivarius or $P$. pentosaceus at $108 \mathrm{CFU} / \mathrm{mL}$, respectively. The tubes were incubated in the air rocking incubator (Thermo Fisher Scientific Inc., Massachusetts, USA) at $37^{\circ} \mathrm{C}$ for $48 \mathrm{~h}$. Five tubes in each treatment were randomly chosen at 12, 24, 36 and $48 \mathrm{~h}$ to determine the ammonia content in medium (Nanjing Jiancheng Bioengineering Institute).

\section{Statistical analysis}

All data were expressed as means \pm standard deviation (SD) and SPSS version 22.0 (SPSS Inc., Chicago, IL, USA) was used to perform an analysis of variance test. $\mathrm{p}<0.05$ was considered significant.

\section{RESULTS}

\section{Identification of $L$. salivarius and $P$. pento- saceus strains}

Using the BLAST sequence analysis services of NCBI, QDPP-CF-01-QDPP-CF-04 were most similar to the sequences of $P$. pentosaceus (KJ477391 and KX262845) with 97\%-98\% sequence identity. QDLS-CF-01-QDLS-CF-02 were most similar to the sequences of L. salivarius (AF089108 and AY112743) with 98\%-99\% sequence identity. Phylogenetic tree and sequence homology analyses (Figure 1) showed that QDPP-CF-01-QDPP-CF-04 were placed in the $P$. pentosaceus branch with $97.8 \%-98.1 \%$ sequence homology and belonged to the species $P$. pentosaceus. QDLS-CF-01-QDLS-CF-02 were placed in the $L$. salivarius branch with $97 \%-98 \%$ sequence homology and belonged to the species $L$. salivarius.

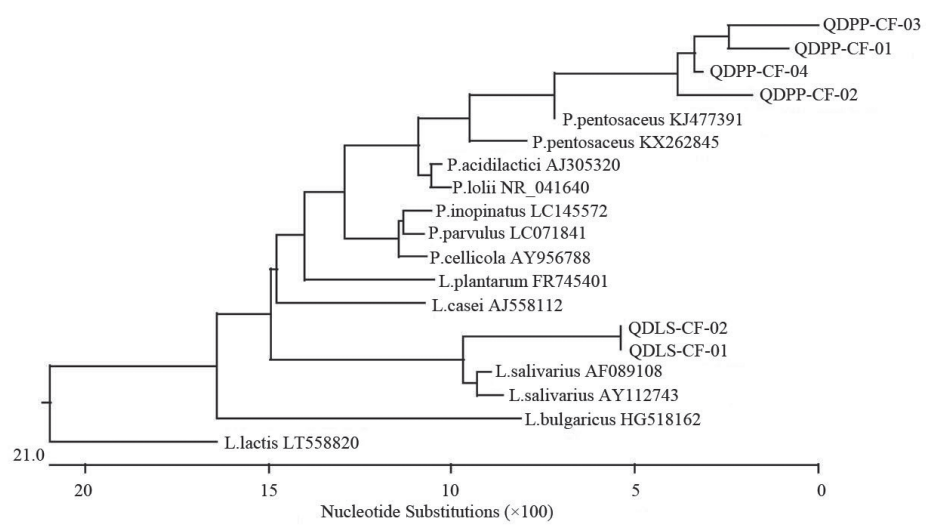

Figure 1 - Phylogenetic relationships among lactic acid bacteria species inferred by a neighbor-joining analysis of a fragment of the partial 16s rRNA sequence. QDLS-CF-01 $\sim$ QDLS-CF-02 and QDPP-CF-01 QDPP-CF-04 are the isolates of lactic acid bacteria from SPF chickens. 
Effects of $L$. salivarius and $P$. pentosaceus supplementation on growth performance of chickens

Compared with the control group, L. salivarius and $P$. pentosaceus supplementation significantly increased BW, ADG, AD-CP, DP and HEYP and decreased FCR $(p<0.05)$ (Table 1$)$, and $L$. salivarius supplementation significantly increased EYP $(p<0.05)$. ADG (d 13-24) and ADG (d 1-24) of $P$. pentosaceus supplementation were significantly higher than that of $L$. salivarius supplementation $(p<0.05)$.

Effects of $L$. salivarius and $P$. pentosaceus supplementation on meat quality of chickens

Compared with the control group, L. salivarius and $P$. pentosaceus supplementation significantly increased fat content in meat $(\mathrm{p}<0.05)$, and fat content of $P$. pentosaceus supplementation was significantly higher than that of $L$. salivarius supplementation $(p<0.05)$ (Table 2 ).

Table 2 - Effects of Lactobacillus salivarius and Pediococcus pentosaceus supplementation on meat quality of chickens

\begin{tabular}{|c|c|c|c|c|}
\hline Group/treatment & & L. salivarius & P. pentosaceus & Control \\
\hline \multirow{5}{*}{ Thigh muscle } & Color & $0.56 \pm 0.04$ & $0.50 \pm 0.03$ & $0.42 \pm 0.12$ \\
\hline & Shear force $(\mathrm{N})$ & $13.0 \pm 0.24$ & $13.6 \pm 0.33$ & $13.3 \pm 0.38$ \\
\hline & Fat content (\%) & $15.3 \pm 0.35^{b}$ & $17.7 \pm 0.48^{a}$ & $12.4 \pm 0.31^{c}$ \\
\hline & Water content (\%) & $64.9 \pm 3.43$ & $63.5 \pm 2.15$ & $65.2 \pm 3.13$ \\
\hline & $\mathrm{pH}$ value & $6.33 \pm 0.09$ & $6.17 \pm 0.08$ & $6.15 \pm 0.09$ \\
\hline \multirow{5}{*}{ Breast muscle } & Color & $0.13 \pm 0.01$ & $0.14 \pm 0.02$ & $0.15 \pm 0.02$ \\
\hline & Shear force $(\mathrm{N})$ & $11.1 \pm 0.42$ & $10.9 \pm 0.34$ & $11.2 \pm 0.24$ \\
\hline & Fat content (\%) & $4.52 \pm 0.53^{b}$ & $6.27 \pm 0.70^{a}$ & $3.62 \pm 0.33^{c}$ \\
\hline & Water content (\%) & $68.2 \pm 2.58$ & $63.9 \pm 3.28$ & $69.5 \pm 3.20$ \\
\hline & $\mathrm{pH}$ value & $5.68 \pm 0.05$ & $5.77 \pm 0.08$ & $5.76 \pm 0.15$ \\
\hline
\end{tabular}

Means in the same line with different letters were significantly different $(p<0.05)$. Data are presented as means $\pm S D$.

Effects of $L$. salivarius and $P$. pentosaceus supplementation on microenvironment of chickens

Compared with the control group, L. salivarius and $P$. pentosaceus supplementation significantly increased the number of Lactobacillus and significantly decreased plasma ammonia content, $\mathrm{pH}$ value and the number of E. coli in ceca, and fecal ammonia emission on days 12 and $24(\mathrm{p}<0.05)$ (Table 3). Fecal ammonia emission of $P$. pentosaceus supplementation was significantly higher than that of $L$. salivarius supplementation on day $24(p<0.05)$.

Effects of $L$. salivarius and $P$. pentosaceus supplementation on ammonia content in medium

Compared with the control group, L. salivarius and $P$. pentosaceus supplementation significantly decreased ammonia content in medium at $12 \mathrm{~h}, 24 \mathrm{~h}, 36 \mathrm{~h}$ and $48 \mathrm{~h}(\mathrm{p}<0.05)$. P. pentosaceus supplementation significantly decreased ammonia content in medium

Table 3 - Effects of Lactobacillus salivarius and Pediococcus pentosaceus supplementation on microenvironment in chicken

\begin{tabular}{llcc}
\hline Items & L. salivarius & P. pentosaceus & Control \\
\hline Plasma ammonia content $(\mathrm{mmol} / \mathrm{L})$ & $75.8 \pm 7.46^{\mathrm{b}}$ & $71.7 \pm 9.27^{\mathrm{b}}$ & $123.5 \pm 9.77^{\mathrm{a}}$ \\
Plasma uric acid $(\mathrm{mg} / \mathrm{L})$ & $34.6 \pm 2.23$ & $35.5 \pm 2.11$ & $32.8 \pm 3.95$ \\
Plasma urea nitrogen $(\mathrm{mmol} / \mathrm{L})$ & $2.26 \pm 0.51$ & $2.71 \pm 0.20$ & $2.54 \pm 0.30$ \\
No. of $E$. coli in ceca $\left(\log _{10} \mathrm{CFU} / \mathrm{g}\right)$ & $7.28 \pm 0.18^{\mathrm{b}}$ & $7.19 \pm 0.31^{\mathrm{b}}$ & $8.20 \pm 0.23^{\mathrm{a}}$ \\
No. of Lactobacillus in ceca $\left(\log _{10} \mathrm{CFU} / \mathrm{g}\right)$ & $8.86 \pm 0.42^{\mathrm{a}}$ & $8.32 \pm 0.22^{\mathrm{a}}$ & $7.80 \pm 0.16^{\mathrm{b}}$ \\
pH value & $5.60 \pm 0.14^{\mathrm{b}}$ & $5.62 \pm 0.14^{\mathrm{b}}$ & $6.14 \pm 0.27^{\mathrm{a}}$ \\
Ammonia emission $(12 \mathrm{~d}, \mathrm{mg} / \mathrm{L})$ & $2.42 \pm 0.15^{\mathrm{b}}$ & $2.62 \pm 0.44^{\mathrm{b}}$ & $7.64 \pm 1.06^{\mathrm{a}}$ \\
Ammonia emission $(24 \mathrm{~d}, \mathrm{mg} / \mathrm{L})$ & $3.89 \pm 0.22^{\mathrm{b}}$ & $2.39 \pm 0.46^{\mathrm{c}}$ & $8.49 \pm 0.16^{\mathrm{a}}$ \\
\hline
\end{tabular}

Means in the same line with different letters were significantly different $(p<0.05)$. Data are presented as means $\pm S D$. 
more than $L$. salivarius supplementation did at $24 \mathrm{~h}$ and $36 \mathrm{~h}(\mathrm{p}<0.05)$ (Figure 2).

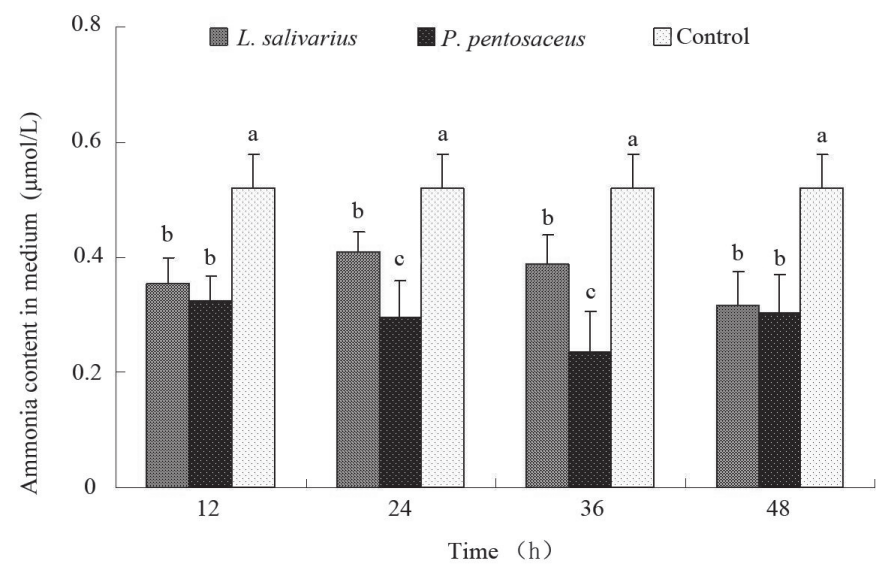

Figure $\mathbf{2}$ - Effects of Lactobacillus salivarius and Pediococcus pentosaceus supplementation on the ammonia content in medium. Data are presented as means \pm SD.

\section{DISCUSSION}

One of the most common methods to isolate and identify novel probiotic strains is phylogenetic and homology analysis of $16 \mathrm{~s}$ rDNA sequences after cultivation in isolation medium (Chen et al., 2013; Tulini et al., 2013; Yanagida et al., 2008). In the present study, six strains of lactic acid bacteria were isolated from the cecal content of SPF chickens on MRS Agar under anaerobic conditions. PCR products of the six strains were sequenced, and nucleotide sequences were most similar to the sequences of $P$. pentosaceus or $L$. salivarius with the highest sequence identity by BLAST sequence analysis and placed in the $P$. pentosaceus branch or the $L$. salivarius branch with the highest sequence homology by phylogenetic and homology analysis of $16 \mathrm{~s}$ rDNA sequence. These results demonstrated that the six strains of lactic acid bacteria isolated from the cecal content of chickens belonged to the species $P$. pentosaceus and $L$. salivarius.

In this study, $L$. salivarius and $P$. pentosaceus supplementation improved growth performance by increasing BW, ADG, DP, AD-CP and HEYP and decreasing FCR of chickens, which is consistent with some previous studies of probiotics. Suo et al. (2012) reported that Lactobacillus plantarum ZJ316 significantly improved pig growth at a dose of $10^{9} \mathrm{CFU} / \mathrm{d}$, and ADG and FCR were better. Foo et al. (2003) reported that feeding Lactobacillus I-UL4 and the metabolites to weaned rats improved growth. Peng et al. (2015) reported that the basal diet supplemented with $2 \times$ $10^{9} \mathrm{CFU} / \mathrm{kg}$ L. plantarum B1 enhanced ADG and FCR in broilers. However, Lähteinen et al. (2014) reported that Lactobacillus brevis supplementation induced a non-significant increase in BW of piglets. Many factors may contribute to the different performance, including the bacterial species and dosage, laboratory animals, and experimental conditions. We considered that the main reason may be that $L$. salivarius and $P$. pentosaceus isolated from chickens were used in the same laboratory animals.

$L$. salivarius and $P$. pentosaceus supplementation significantly improved in vivo and in vitro microenvironment by increasing the number of Lactobacillus in ceca and decreasing plasma ammonia content, $\mathrm{pH}$ value and the number of $E$. coli in ceca, and fecal ammonia emission. Many studies have reported that Lactobacillus spp. and Bacillus spp. had better effects on controlling ammonia emission and intestinal flora (Biagi et al., 2007; Jeong and Kim, 2008; Zhang et al., 2013; Ahmed et al., 2014; Altaher et al., 2015). Zhang et al. (2013) considered that ammonia emission was related to nutrient utilization and the intestinal microflora ecosystem, and some probiotics might reduce ammonia emission by improving enzyme activity and nitrogen utilization. Otherwise, some probiotics supplementation will result in a significant reduction in ammonia emission without any accompanying improvement in nitrogen digestibility. We considered that $L$. salivarius and $P$. pentosaceus improved the microenvironment in the following aspects. (i) L. salivarius and P. pentosaceus might secrete some antimicrobial compounds, such as organic acids, hydrogen peroxide and bacteriocins, which might have a potential modulating effect on the intestinal microflora and pathogen inhibition and decrease $\mathrm{pH}$ value in ceca. These compounds are unsuitable for the activity and proliferation of pathogens such as $E$. coli, but suitable for $L$. salivarius and $P$. pentosaceus populations. (ii) $L$. salivarius and $P$. pentosaceus could produce high levels of ammonia nitrogen and then increase AD-CP, which might be one of the reasons for reducing ammonia level. Moreover, the in vitro experiment showed that $L$. salivarius and $P$. pentosaceus significantly decreased the ammonia level in the medium. Therefore, we considered that $L$. salivarius and $P$. pentosaceus had a capacity for ammonia nitrogen fixation and enhanced ammonia nitrogen transformation. Further study is needed to elucidate other possible mechanisms of reducing ammonia emission.

$L$. salivarius and $P$. pentosaceus supplementation could improve meat quality; especially increasing the fat content in muscles. Many studies have demonstrated the potential effects of other probiotics on meat 
quality, including Clostridium butyricum in pigs (Meng et al., 2010) and Bacillus coagulans (Zhou et al., 2010), Bacillus subtilis (Fan et al., 2013; Park and Kim, 2014), Enterococcus faecium (Zheng et al., 2014), L. plantarum (Loh et al., 2013) and Rhodopseudomonas palustris (Xu et al., 2014) in chickens. These results showed a lot of different indicators of the effects of probiotics on meat quality (Suo et al., 2012). Zhang et al. (2015) considered that the shear force of raw drumstick decreased in the whole yeast treatment relative to the controls. Zhou et al. (2010) demonstrated that $B$. coagulans probiotic affected meat quality by decreasing the drip loss of broiler breast meats of Guangxi Yellow chickens. Meng et al. (2010) demonstrated that the probiotic treatment groups had increased meat color scores, marbling scores, and redness values in growingfinishing pigs. Until now, the functioning mechanism of probiotics (especially Lactobacillus) on meat quality (fat content in the study) remains unclear, although some investigators have attributed the effects of probiotics on meat quality to the role of bacteriocins or extracts (Zheng et al., 2014). We considered that further study of the effects of $L$. salivarius and $P$. pentosaceus on meat quality was required.

In conclusion, the present study demonstrates that $L$. salivarius and $P$. pentosaceus improve growth performance, meat quality, ammonia emission and intestinal environment in chickens and are promising probiotic properties.

\section{ACKNOWLEDGMENTS}

We are grateful to our laboratory technicians for their assistance with the experimental animals. This study was supported by Shandong modern agricultural industry technology system poultry innovation team projects (No. SDAIT-13-011-07) and National Natural Science Foundation of China (No. 31001093).

\section{REFERENCES}

Ahmed ST, Islam M, Mun HS, Sim HJ, Kim YJ, Yang CJ. Effects of Bacillus amyloliquefaciens as a probiotic strain on growth performance, cecal microflora, and fecal noxious gas emissions of broiler chickens. Poultry Science 2014;93:1963-1971.

Altaher YW, Jahromi MF, Ebrahim R, Zulkifli I, Liang JB. Lactobacillus Pentosus Ita23 and L. Acidipiscis Ita44 enhance feed conversion efficiency and beneficial gut microbiota in broiler chickens. Brazilian Journal of Poultry Science 2015;17(2):159-164.

Biagi G, Cipollini I, Pompei A, Zaghini G, Matteuzzi D. Effect of a Lactobacillus animalis strain on composition and metabolism of the intestinal microflora in adult dogs. Veterinary Microbiology 2007;124:160-165.
Carraro L, Maifreni M, Bartolomeoli I, Martino ME, Novelli E, Frigo F, et al. Comparison of culture-dependent and -independent methods for bacterial community monitoring during Montasio cheese manufacturing. Research of Microbiology 2011;162:231-239.

Chen YS, Liou MS, Ji SH, Yu CR, Pan SF, Yanagida F. Isolation and characterization of lactic acid bacteria from yan-tsai-shin (fermented broccoli stems), a traditional fermented food in Taiwan. Journal of Applied Microbiology 2013;115(1):125-132.

Fan Y, Zhao L, Ma Q, Li X, Shi H, Zhou T, et al. Effects of Bacillus subtilis ANSB060 on growth performance, meat quality and aflatoxin residues in broilers fed moldy peanut meal naturally contaminated with aflatoxins. Food Chemistry Toxicology 2013;59:748-753.

Foo HL, Loh TC, Lai PW, Lim YZ, Kufli CN, Rusul G. Effects of adding L.plantaruml-UL4 metabolites in drinking water of rats. Pakistan Journal of Nutrition 2003;2:283-288.

Jeong JS, Kim IH. Effect of Bacillus subtilis C-3102 spores as a probiotic feed supplement on growth performance, noxious gas emission, and intestinal microflora in broilers. Poultry Science 2014;93:3097-3103.

Kiran F, Mokrani M, Ozlem O. Effect of encapsulation on viability of Pediococcus pentosaceus OZF during its passage through the gastrointestinal tract model. Current Microbiology 2015;71:95-105.

Lähteinen T, Lindholm A, Rinttilä T, Junnikkala S, Kant R, Pietilä TE, et al. Effect of Lactobacillus brevis ATCC 8287 as a feeding supplement on the performance and immune function of piglets. Veterinary Immunology and Immunopathology 2014;158:14-25.

Loh TC, Thanh NT, Foo HL, Hair-Bejo M. Effects of feeding metabolite combinations from lactobacillus plantarum on plasma and breast meat lipids in Broiler Chickens. Brazilian Journal of Poultry Science 2013;15(4):307-316

Meng QW, Yan L, Ao X, Zhou TX, Wang JP, Lee JH, et al. Influence of probiotics in different energy and nutrient density diets on growth performance, nutrient digestibility, meat quality, and blood characteristics in growing-finishing pigs. Journal of Animal Science 2010;88:3320-3326.

Mookiah S, Sieo CC, Ramasamy K, Abdullah N, Ho YW. Effects of dietary prebiotics, probiotic and synbiotics on performance, caecal bacterial populations and caecal fermentation concentrations of broiler chickens. Journal of the Science of Food and Agriculture 2014;94:341-348.

Nagpal R, Kumar A, Kumar M, Behare PV, Jain S, Yadav H. Probiotics, their health benefits and applications for developing healthier foods: a review. FEMS Microbiology Letter 2012;334:1-15.

National Research Council. Nutrient requirements of poultry. 9th ed Washington (DC): National Academy of Science; 1994.

Noujaim JC, Andreatti Filho RL, Lima ET, de Okamoto AS. Detection of CD4+ and CD8+ lymphocytes in the intestine of broiler chicks treated with Lactobacillus spp. and challenged with Salmonella enterica serovar Enteritidis. Brazilian Journal of Poultry Science 2009;11(3):187-193.

Okamoto AS, Andreatti Filho RL, Lima ET, Pereira REP, Menconi A, Rocha TS, et al. Immunological evaluation of the intestinal mucosa of broiler chicks treated with Lactobacillus Spp. and challenged with Salmonella Enteritidis. Brazilian Journal of Poultry Science 2007;9(4):259-262.

Osmanagaoglu O, Kiran F, Ataoglu H. Evaluation of in vitro probiotic potential of Pediococcus pentosaceus OZF isolated from human breast milk. Probiotics and Antimicrobial Proteins 2010;2:162-174.

Park JH, Kim IH. Supplemental effect of probiotic Bacillus subtilis B2A on productivity, organ weight, intestinal Salmonella microflora, and breast meat quality of growing broiler chicks. Poultry Science 2014;93:20542059. 
Peng Q, Zeng XF, Zhu JL, Wang S, Liu XT, Hou CL, et al. Effects of dietary Lactobacillus plantarum B1 on growth performance, intestinal microbiota, and short chain fatty acid profiles in broiler chickens. Poultry Science 2016;95:893-900.

Suo $C$, Yin Y, Wang X, Lou X, Song D, Wang X, et al. Effects of lactobacillus plantarum ZJ316 on pig growth and pork quality. BMC Veterinary Research 2012;8:89.

Thiex, NJ, Anderson S, Gildemeister B. Crude fat, hexanes extraction, in feed, cereal grain, and forage (Randall/Soxtec/submersion method): collaborative study. Journal of AOAC International 2003;86:899-908.

Tulini FL, Winkelströter LK, De Martinis EC. Identification and evaluation of the probiotic potential of Lactobacillus paraplantarum FT259, a bacteriocinogenic strain isolated from Brazilian semi-hard artisanal cheese. Anaerobe 2013;22:57-63.

Vidhyasagar $\mathrm{V}$, Jeevaratnam $\mathrm{K}$. Bacteriocin activity against various pathogens produced by Pediococcus pentosaceus VJ13 isolated from Idly batter. Journal of Chromatography B-Analytical Technologies in the Biomedical and Life Sciences 2013;27:1497-1502.

Xie J, Yu Q, Nie S, Fan S, Xiong T, Xie M. Effects of Lactobacillus plantarum NCU116 on intestine mucosal immunity in immunosuppressed mice. Journal of Agricultural and Food Chemistry 2015;63:10914-10920.

Xu QQ, Yan H, Liu XL, Lv L, Yin CH, Wang P. Growth performance and meat quality of broiler chickens supplemented with Rhodopseudomonas palustris in drinking water. British Poultry Science 2014;55:360-366.
Yanagida F, Srionnual S, Chen YS. Isolation and characteristics of lactic acid bacteria from koshu vineyards in Japan. Letters in Applied Microbiology 2008:47(2):134-139.

Zheng A, Luo J, Meng K, Li J, Zhang S, Li K, et al. Proteome changes underpin improved meat quality and yield of chickens (Gallus gallus) fed the probiotic Enterococcus faecium. BMC Genomics 2014;15:1167.

Zhang AW, Lee BD, Lee SK, Lee KW, An GH, Song KB, et al. Effects of yeast (Saccharomyces cerevisiae) cell components on growth performance, meat quality, and ileal mucosa development of broiler chicks. Poultry Science 2005;84:1015-1021.

Zhang J, Deng J, Wang Z, Che C, Li Y, Yang Q. Modulatory effects of Lactobacillus salivarius on intestinal mucosal immunity of piglets. Current Microbiology 2011;62:1623-1631.

Zhang M, Fan X, Fang B, Ren V, Zhu C, Zhu J, et al. Effects of Lactobacillus salivarius Ren on cancer prevention and intestinal microbiota in 1 , 2-dimethylhydrazine-induced rat model. Journal of Microbiology 2015;53:398-405

Zhou X, Wang Y, Gu Q, Li W. Effect of dietary probiotic, Bacillus coagulans, on growth performance, chemical composition, and meat quality of Guangxi Yellow chicken. Poultry Science 2010;89:588-593.

Zhang ZF, Kim IH. Effects of probiotic supplementation in different energy and nutrient density diets on performance, egg quality, excreta microflora, excreta noxious gas emission, and serum cholesterol concentrations in laying hens. Journal of Animal Science 2013;91:4781-4787. 
